\title{
Draw Your Own Story: Paper and Pencil Interactive Storytelling
}

\author{
Edirlei Soares de Lima ${ }^{1}$, Bruno Feijó ${ }^{1}$, Simone Barbosa ${ }^{1}$, Antonio L. Furtado ${ }^{1}$, \\ Angelo Ciarlini ${ }^{2}$, and Cesar Pozzer ${ }^{3}$ \\ ${ }^{1}$ PUC-Rio - Departamento de Informática, Rio de Janeiro - Brazil \\ ${ }^{2}$ UNIRIO - Departamento de Informática Aplicada, Rio de Janeiro - Brazil \\ ${ }^{3}$ UFSM - Departamento de Eletrônica e Computação, Santa Maria - Brazil \\ \{elima, bfeijo, simone, furtado\} ainf.puc-rio.br, \\ angelo.ciarlini@uniriotec.br, pozzer@inf.ufsm.br
}

\begin{abstract}
In this paper, we present a storytelling system able to dramatize interactive narratives in augmented reality over a conventional sheet of paper. The system allows users to freely interact with virtual characters by sketching objects on the paper. Users interacting with the system can indirectly affect the characters' decisions, even to the point of radically subverting the storyline.
\end{abstract}

Keywords: Interactive Storytelling, Augmented Reality, Sketch-Based Interface.

\section{Introduction}

Since from times immemorial, humans have been using hand drawings, sometimes rather crude but good enough to convey a personal touch, as a visual complement to oral storytelling. Currently, stories are told through several types of media (books, movies, games) and, with the advent of new technologies, digital storytelling is becoming increasingly interactive. How to combine visualization and interaction while a story is being created is even today an active field of research. Though it seems convenient to equip storytelling systems with user interfaces inspired on video game interfaces, there is a risk that such interfaces may restrict user immersion and creativity.

HCI researchers have been focusing on immersive interfaces over the last fifteen years, from different viewpoints: multimodal interfaces [19][20], virtual reality [19], and augmented reality (AR) [17][18]. However, few immersive systems are devoted to interactive storytelling. Moreover, these few works require special devices, such as CAVE-like immersive displays [22] and see-through head-worn displays [21]. Complex devices installations make the immersion experience less natural and creative. An interesting way of exploring the user's creativity and natural engagement in interactive storytelling is the use of sketch-based interfaces.

Sketch-based interaction has been used in engineering, education and 3D modeling (see the special issue on this subject elsewhere [23]), and it is a permanent research topic since Ivan Sutherland proposed his famous SketchPad system [24] in the sixties. Those systems use special input devices (such as tablets) or the projection display. 
Augmented reality systems using sketch-based interaction and simple paper and pencil have also been proposed [25][1]. However, the development of AR systems for interactive storytelling is still an open research topic.

In this paper, we explore the use of an augmented reality visualization interface combined with a sketch-based interaction interface. We present a storytelling system able to dramatize interactive narratives in augmented reality over a conventional sheet of paper. The system allows users to freely interact with virtual characters by sketching objects on the paper. The system recognizes the hand-drawn sketches and converts the drawings into virtual objects in the 3D story world.

The paper is organized as follows. Section 2 describes related works. Section 3 presents the architecture and implementation of the paper and pencil interactive storytelling system. Section 4 describes a simple application, together with a preliminary user evaluation. Section 5 contains the concluding remarks.

\section{Related Work}

The use of hand-drawn sketches in an interactive narrative environment is discussed by Vogelsang and Signer [2]. Their system is dependent upon a special pen, known as the Anoto Digital Pen ${ }^{1}$ which, when used on a special paper, is capable of recording the user strokes and sending the drawing information to a computer. The idea of transferring paper drawings to a virtual world was explored by Kuka et al. [3]. Their system is also based on the Anoto Digital Pen technology. A combination of mixed reality and collaborative environment is found in Bayon et al. [4]. The authors present a storytelling environment where a variety of devices (PDAs, scanners, bar codes and a large screen display) are used by children to tell stories.

The use of mixed reality environments in the context of interactive narratives has also been the focus of some research projects. Dow et al. [5] present an augmented reality version of the desktop-based interactive drama Façade [6]. With a similar approach, Cavazza et al. [7] present an interactive storytelling application that captures the user's video image and inserts him/her in a world populated by virtual actors. Users are able to interact with the virtual actors using body gestures and natural language speech. While Dow et al. [5] bring the virtual characters to the real world, Cavazza et al. [7] place the user inside the virtual world. Zhou et al. [16] explore the use of tangible cubes as interaction interface for mixed reality interactive storytelling.

In the gaming context, Hagbi et al. [1] explore the use of hand-drawn sketches as a content-authoring tool for augmented reality games. A similar approach is used by Huynh et al. [8]. Leitner et al. [9] explore the interaction between real world objects and virtual objects in board games.

None of the above-mentioned works appears to combine all the characteristics of the system proposed in the present work, that is: a paper and pencil interactive storytelling tool with a sketch-based AR interface that allows an easy and more natural way of interfering in the ongoing story.

${ }^{1}$ http : / /www . anoto. com 


\section{Paper and Pencil Interactive Storytelling}

The proposed system (Figure 1) is composed of a computer equipped with a conventional webcam, an ordinary sheet of paper with a fiducial marker printed on it, and a common pencil.

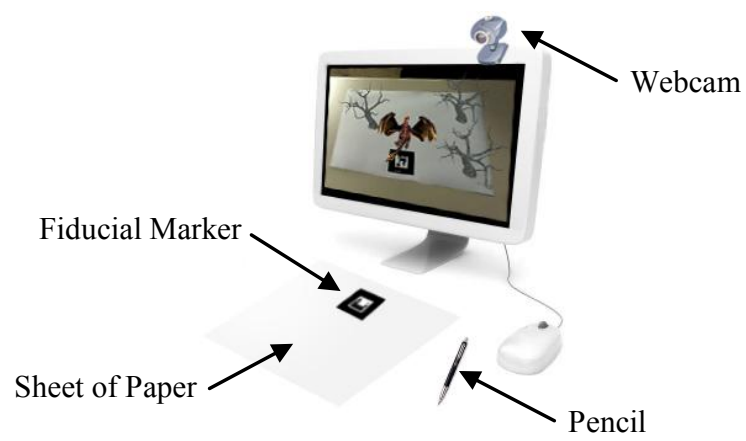

Fig. 1. The simple environment of the proposed system

In the system, stories are graphically represented in augmented reality over the paper, which creates the illusion that the sheet of paper is a virtual world populated by virtual characters. The entire world may comprise several sheets of paper, each one representing a different location in the virtual world. Users can switch between places by changing the paper shown to the camera or by pointing the camera to other sheets of paper. They can distinguish the places represented by the various pages based on their respective fiducial markers.

Users act as gods of the virtual world, in a way reminiscent of the deus ex machina of classical theater. For example, a hero may not have enough strength to slay the villain with his bare hands, but if the user draws a sword close to the hero's position in the paper, the sword will be transferred to the virtual world and taken by the hero, who will now be able to defeat the villain.

The paper and pencil interactive storytelling system is composed of three main modules: the story planner, the sketch recognition interface and the augmented reality dramatization system (Figure 2). The story planner handles the actions of several virtual autonomous characters, each one introduced with predefined goals, whose behavior may however be redirected via user interactions. The sketch recognition system consists of a support vector machine classifier trained to recognize a set of hand-drawn sketches produced by the users on the sheet of paper, which were captured by the camera. The augmented reality dramatization system controls and renders the virtual world superimposed over the real world objects, creating a mixed reality environment. If a fiducial marker is found on the image, the system renders the virtual world objects and characters according to the virtual location identified by the marker.

The parallel architecture of the system is important to guarantee that there will be no noticeable delays in the rendering process - which is currently limited to 30 frames per second, due to the camera capture speed. Since the recognition of user sketches is the most expensive process in the system, it must be executed in a separate thread, so that the system is able to render the output images effectively in real-time. 


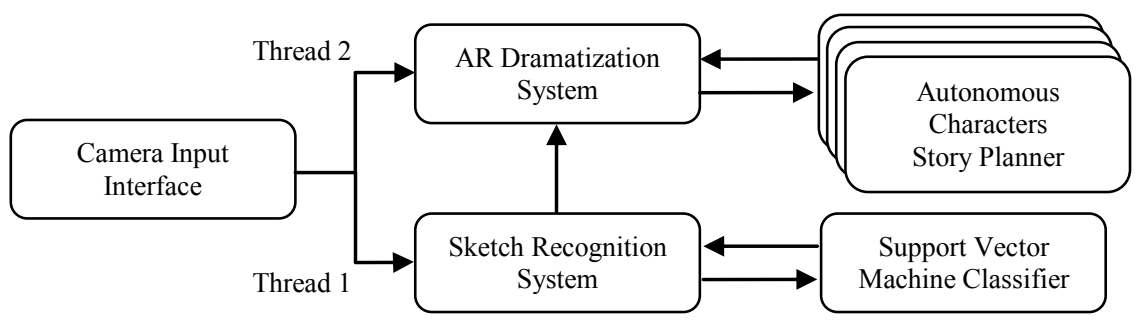

Fig. 2. Parallel system architecture

\subsection{Story Planner}

Interactive storytelling systems can follow three basic approaches: plot-based [27], character-based [30], or hybrid approach [26]. In this paper, we add a layer on top of a nondeterministic hybrid approach to interactive storytelling found in some of our previous works [27][28][29]. In this approach, a nondeterministic planning algorithm generates a plan in which events allow characters to try achieving goals without necessarily succeeding [29]. Furthermore, events can be specified by nondeterministic automata [28], in which the arcs represent short episodes that we call "actions". In the present paper, we propose an interactive layer that can represent an action in those automata. As the actions are nondeterministic, the interactions that occur via this layer can influence the rest of the story.

In the presented layer, the story evolves towards surprising outcomes depending on emotional and physical states that characters can attain as a result of the user interventions over a sheet of paper. The example implemented in the prototype corresponds to a short story within a swords and dragons genre. In this story, a villain (dragon) kidnaps a narcissistic princess, who can easily get depressed, and a brave knight tries to rescue her.

The emotional, physical, and social attributes of the characters are modeled as a multi-character network (Figure 3), where nodes represent characters and bidirectional arcs define affection relationships in the social environment of the story. Each node has the name of the character and the values of the emotional/physical attributes. Affections are not reciprocal, that is affection $(i, j)$ is not necessarily equal to affection $(j, i)$, except when there is a self-affection situation. Affection values vary within the interval $[-10,10]$.

The emotional model adopted by our planner uses the six emotions proposed by Ekman and Friesen [10], but we consider them lying on six emotion axis with negative and positive sides that represent opposite emotions: [calmness, anger], [liking, disgust], [confidence, fear], [joy, sadness], [cheeriness, sorrow], and [anticipation, surprise]. The values in each axis are numbers within the interval [-10, 10]. In this model, sorrow is not a synonym of sadness, but a sense of loss or a sense of guilt and remorse. For the sake of simplicity, we refer to an axis by the name of its positive side. The sign $(-$ or + ) does not mean destructive or constructive emotions, but a connotation of drama impact and opposite states. 


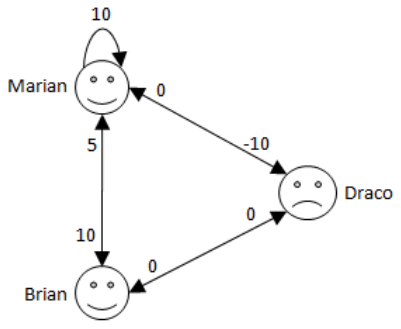

\begin{tabular}{|c|c|c|c|}
\hline Name: Brian & Name:Draco & Name: Marian & \\
\hline Anger:-1 & Anger: 8 & Anger:-8 & \\
\hline Disgust: -4 & Disgust: 7 & Disgust: 2 & \\
\hline Fear: 3 & Fear:-8 & Fear: -4 & Emotional \\
\hline Sadness: 1 & Sadness: 7 & Sadness: 2 & Attributes \\
\hline Sorrow:-3 & Sorrow:-9 & Sorrow:-3 & \\
\hline Surprise:-1 & Surprise: 3 & Surprise:-4 & \\
\hline Strength: 2 & Strength:7 & Strength: -10 & Physical \\
\hline Defense: 2 & Defense: 5 & Defense:-9 & Attributes \\
\hline
\end{tabular}

Fig. 3. The proposed multi-character network

In the proposed model, emotions can be combined to form a new emotion, for instance: love $=$ joy + liking + confidence. Also, we can refer to extreme values on an axis as being special emotions, e.g.: grief = very high levels of sadness and ecstasy = very high levels of joy (that is, very low levels of sadness).

The story planner used by the paper-and-pencil interactive layer is defined by general rules and production rules. General rules express general knowledge about the genre, such as "if a person is not at home he/she will be unprotected". For example: $\forall X$ currentPlace $(X) \neq$ home $(X) \rightarrow \sim \operatorname{protected}(X)$, where $\sim$ denotes negation.

Production rules concern actions and have the following form:

\section{CONDITIONS $\rightarrow$ actions(CONCRETE_ACTIONS, ATTRIBUTE_CHANGES)}

where CONDITIONS is a conjunction of observed facts; CONCRETE_ACTIONS is a list of concrete actions, such as go, take, hit, and kidnap; and ATTRIBUTE_CHANGES is a list of increments and decrements to the indicated characters' attributes using the operators add(X,Attribute,Increment) or $\operatorname{addr}(X, Y$, Attribute,Increment), e.g. add(marian,sadness,9) increments Marian's current value of sadness by 9 and addr(marian,brian,affection,5) increments Marian's affection to Brian by 5 . In the proposed model, we work with attribute operators that return the current level of a specific attribute for a specific character $X$. These operators use the same terminology of the multi-character network, e.g. sadness $(X)$ and affection $(X, Y)$. Also the model has an important operator that confirms if a specific Object drawn by the user can be seen by a character $X$ : cansee $(X, O b j e c t)$. For example, if the user draws a mirror on the paper close to princess Marian, cansee(marian,mirror) will be true. Users interacting with the system can indirectly affect the characters' decisions, even to the point of radically subverting the storyline. The following sentences are examples of production rules used in the prototype:

(a) Bored princesses always become sad:

$\forall X \operatorname{princess}(X) \wedge \operatorname{protected}(X) \wedge \operatorname{surprise}(X)<-2 \rightarrow \operatorname{actions}([],[\operatorname{add}(X, \operatorname{sadness}, 9)])$

(b) Drawing objects on paper (detected by cansee) causes changes in attribute levels:

$\forall X$ princess $(X) \wedge$ free $(X) \wedge$ cansee $(X$, mirror $)$

$\rightarrow \operatorname{actions}([\operatorname{go}(X$, mirror $)$, take $(X$, mirror $)],[\operatorname{add}(X$, surprise, 3$), \operatorname{add}(X, \operatorname{sadness},-3)])$ 
(c) Actions can be more complex than simple movements, such as the one that defines the act of kidnapping:

$\forall X \forall Y$ villain $(X) \wedge$ affection $(X, Y)<-8 \wedge \sim \operatorname{protected}(Y) \wedge$ free $(Y)$

$\rightarrow \operatorname{actions}([\operatorname{go}(X, \operatorname{currentPlace}(Y)), \operatorname{hit}(X, Y), \operatorname{kidnap}(X, Y), \operatorname{go}(X, \operatorname{home}(X)],[])$

Our previous works [27][28][29] use Constraint Logic Programming to specify rules used in plot generation and dramatization control at a higher level. In this work, however, actions occur at a lower level and we decided to use a $\mathrm{C}++$ forward chaining procedure here, due to performance reasons. In the present work, we implement a story loop with a fixed time step (15 seconds) to update the characters' states. In every story loop all the rules are executed again. When the user interferes in the story, the states are updated as soon as a new object is created on the sheet of paper.

\subsection{Hand-Drawn Sketches Recognition}

The process of recognizing hand-drawn sketches can be divided into a pre-processing phase and a recognition phase.

\subsubsection{Pre-Processing Phase}

The objective of the pre-processing phase is to eliminate the background of the input image and highlight the drawing forms. The approach used in this work to segment the image and remove the background is based on the application of five increasing threshold levels and a canny edge detector over the input image. This process generates six new images containing the candidate drawings. The use of several threshold levels is important to ensure that the drawings may be identified by the system even with different illumination conditions.

The threshold levels and the canny edge detector reduce the number of objects on the image, but are still not enough to clear all the background. To completely clear the background, the system uses the paper rectangular border as a boundary to ignore all the objects that are outside it. The sheet of paper is recognized as the largest rectangle on the image. The augmented reality marker, located inside of the paper, is also removed at the pre-processing phase to avoid being classified as a user drawing. The segmentation process is illustrated in Figure 4.

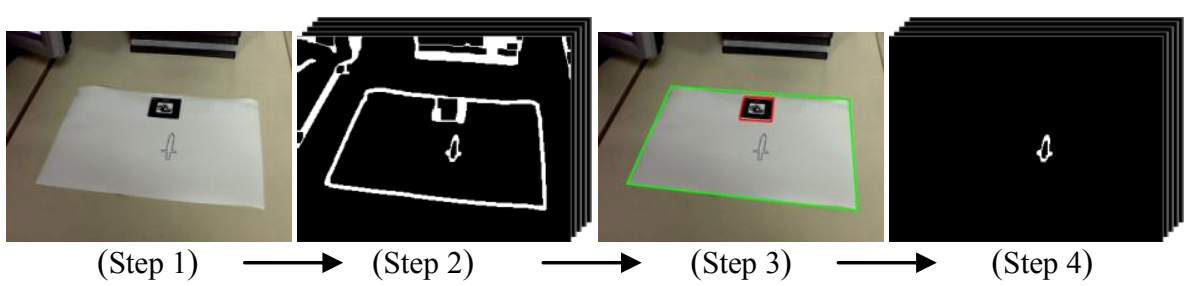

Fig. 4. Segmentation process. (Step 1) input frame captured by the camera; (Step 2) segmented images; (Step 3) detection of the paper rectangle and the marker; (Step 4) set of candidate drawings. 


\subsubsection{Recognition Phase}

In the recognition phase, the sketches (previously segmented during the preprocessing phase) are classified according to a predefined set of drawings. To perform this classification, the system uses a support vector machine (SVM) classifier trained with structural features to classify hand-drawn sketches. SVM [11] has proved effective in many real-world applications, such as in systems for detecting microcalcifications in medical images [12], automatic hierarchical document categorization [13], and 3D camera control [14].

The SVM structure is composed of the output classes (the vocabulary of handdrawn sketches understood by the classifier) and the features vector (numerical features characterizing the hand-drawn sketches). The classifier uses the features (in a training phase) to create a pattern that classifies unknown input features vectors in one of the output classes (prediction process).

The features vector adopted in the implementation of our classifier is composed of seven moment invariants, which are extracted from the candidate drawings found on the segmented images. The Hu descriptors, proposed by $\mathrm{Hu}$ [15], are based on nonorthogonalised central moments that are invariant to image rotation, translation, and scale. This invariance requirement is essential for allowing the classifier to recognize the drawings from different camera positions and angles. The combination of those seven $\mathrm{Hu}$ descriptors uniquely characterizes a specific pattern or shape.

Before using the SVM to recognize hand-drawn sketches, the classifier must be trained. The training process consists of capturing (from different angles) several images of hand-drawn sketches, and then processing these images to segment the background and extract the drawing features used by the SVM.

The recognition process can be summarized as follows: (1) Extraction of the contours from each candidate drawing found in pre-processed images; (2) Extraction of the features used by the SVM classifier from each candidate drawing; (3) Filtration of the candidate drawings that have too small areas or a number of vertices outside the range defined for the known drawings; (4) Classification of the candidate drawings using the SVM classifier; (5) Analysis of the SVM output to identify the drawing recognized by the classifier.

For each image frame captured by the camera, the system classifies the hand-drawn sketches found at all segmentation levels resulting from the pre-processing phase. In this way, the system has the classification of the same drawing in different segmentation conditions. Based on these results, the algorithm can search for the bestfitting class. A voting approach is adopted to choose the final classification of the drawing.

\subsection{Augmented Reality Dramatization}

The augmented reality dramatization system uses the ARToolKit Library ${ }^{2}$, which encapsulates functions to calculate the position of the real camera based on the size and orientation of physical fiducial markers. Each marker has a distinctive pattern and is associated with a specific location of the virtual world (Figure 5).

${ }^{2}$ http: / /www.hitl.washington. edu/artoolkit/ 

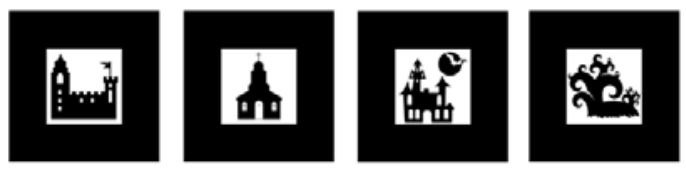

Fig. 5. Fiducial markers used by the system to compute the position of the virtual camera according to the real camera. The maker image is also used to identify the virtual places.

The dramatization system represents the characters of the stories through animated 3D models that can walk freely across the scenarios displayed over the sheets of paper. The virtual characters who are in the location where an object was created are informed about the presence of the new object. The planning system then chooses the appropriate actions for the characters according to the observed situation. The user interaction process is illustrated in Figure 6.

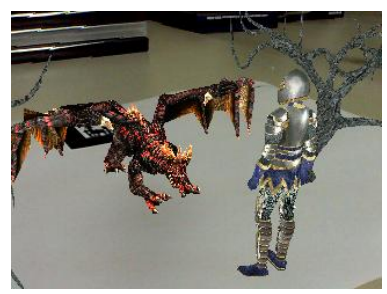

(a)

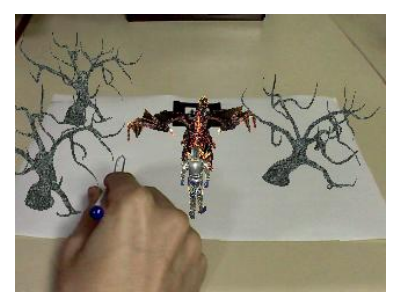

(b)

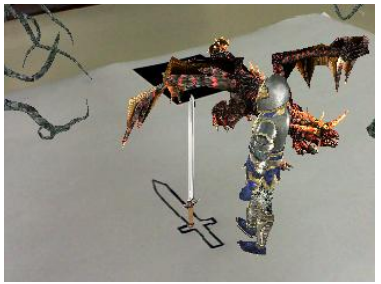

(c)

Fig. 6. User Interaction. Image (a) shows a scene being dramatized in a virtual place; image $(b)$ shows the user sketching an object; and finally, image $(c)$ shows the sketched object (sword, in this case) coming to life in the augmented reality environment.

\section{Application and Evaluation}

The prototype application developed to test our system is based on an overly simplified "Swords and Dragons" genre. The virtual world is populated by three main characters: the charming, narcissistic, and lonely princess Marian, who lives under strict protection at a palace; a brave young knight, sir Brian, in love with the princess; and the evil dragon, Draco, constantly waiting for a chance to kidnap the princess. The virtual world is composed of four places: the princess's palace, the dragon forest, a church and the forest where the wandering hero dwells. Users are able to interact with the virtual characters by sketching on a paper. For the prototype application, the following six items are recognizable, whose presence can somehow affect the storyline: a "hand mirror", which may divert the princess's eyes while increasing her level of joy; a "sword", which Brian would gladly wield to supplement his bodily strength; a "shield", adequate for the hero's defense; a "magic stone", that can dangerously increase Draco's strength; a "rat", which can serve either to distract the dragon's attention, or to scare princess Marian; and a "poison bottle", a possible inducement to Marian or Brian to commit suicide in desperate situations. The recognizable sketches used in the prototype are illustrated in Figure 7. 


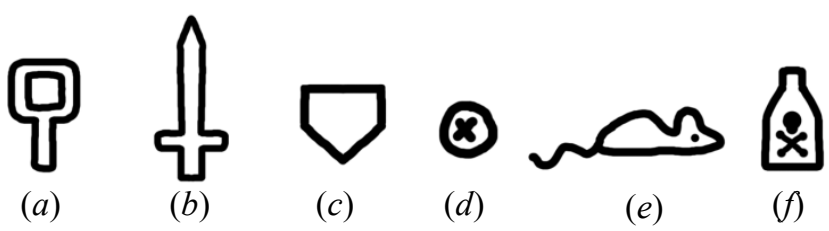

Fig. 7. Recognizable sketches. (a) hand mirror; $(b)$ sword; $(c)$ shield; $(d)$ magic stone; $(e)$ rat; (f) poison bottle.

The prototype application is able to generate a considerable number of diversified stories under the stimulus of the user interactions. For example: in more conventional stories, the princess is kidnapped by the dragon and then saved by the hero who kills the dragon; in stories with a not so happy ending, the hero is defeated by the dragon; and in others with a darker outcome, the dragon kills the princess, or she commits suicide. But the dragon's participation is not really indispensable to cause misfortune. One story was generated wherein the princess, frightened by the rat, broke her mirror, whereupon she became so distraught that she drank the proffered poison.

To evaluate our system, we performed two tests: a technical test to check the performance and accuracy of the system, and then a user evaluation test to check the system's usability from a Human-Computer Interaction (HCI) perspective. The following sections describe these tests.

\subsection{Technical Evaluation}

The technical evaluation concerns the accuracy and the real-time performance of the system. The tests were mainly focused on sketch recognition, which constitutes the most expensive process and includes a machine-learning method that is not guaranteed to provide correct answers at all times.

The evaluation of the sketch recognition system was based on two experiments: (1) the recognition rate test, to check the accuracy of the predicted sketches; and (2) the performance test, to check the time needed to process the input frames and recognize the hand-drawn sketches.

For the recognition rate test, we utilized a collection of 250 pictures (captured by a conventional webcam from different angles), using training datasets ranging from 100 to 300 samples. In this test, the classifier was trained to recognize 6 classes of sketches. The computed average recognition rate is shown in Table 1.

To evaluate the performance of the sketch recognition system, we again utilized a collection of 250 pictures, and calculated the average time necessary to perform the pre-processing and the classification of the sketches. The computer used to run the experiments was an Intel Core i7 2.66 GHZ CPU, 8 GB of RAM using a single core to process the algorithms. Table 1 shows the result of the performance test, with training datasets ranging from 100 to 300 samples. 
Table 1. Recognition rate and performance test with training datasets ranging from 100 to 300 samples

\begin{tabular}{|c|c|c|c|c|c|}
\hline Training Samples & $\mathbf{1 0 0}$ & $\mathbf{1 5 0}$ & $\mathbf{2 0 0}$ & $\mathbf{2 5 0}$ & $\mathbf{3 0 0}$ \\
\hline Recognition Rate & $92.1 \%$ & $92.8 \%$ & $93.4 \%$ & $93.4 \%$ & $93.8 \%$ \\
\hline Recognition Time $(\boldsymbol{m s})$ & 83.6 & 81.2 & 84.4 & 84.7 & 85.1 \\
\hline
\end{tabular}

Analyzing the test results, it seems fair to conclude that the classifier ensures high recognition rates without sacrificing the system's performance.

\subsection{User Evaluation}

We have conducted a preliminary evaluation with six participants, four male and two female, all between 20 and 26 years old, with diverse backgrounds: a cinema professional, an undergraduate student in Media Studies, a graduate student and two undergraduate students in Computer Science, and an undergraduate student in Fine Arts with some experience in Computer Science.

We asked participants to interact with the story using our system, including objects and changing scenes to influence the story unfolding as they wished. They were asked to interact both with our system $(\mathrm{S})$ and with a modified version of it $(\mathrm{M})$ that used menus to include objects in the scene instead of sketching. In order to reduce learning effects, half of the participants used S first, and the other half used $\mathrm{M}$ first.

After using each version, the participants filled out a questionnaire with 21 questions about their motivation to change the story, their understanding of how to do so, the effort to do so, and how immersed they were in the story creation, as reflected in their awareness of their surroundings during their interaction with the system. After having interacted with both systems, the participants were interviewed about their preferences and what they would like to see improved in the sketching version.

Figure 8 summarizes the results of the questionnaire. As for the interviews, all participants stated they preferred to interact with the sketch-based version, because it was more interesting, attractive, exciting, innovative, caught their attention more, and allowed them to explore their creativity, despite the slightly increased effort, mostly due to some limitations of the recognition algorithm. They thought the menu-based version was too simplified, easier, but less motivating.

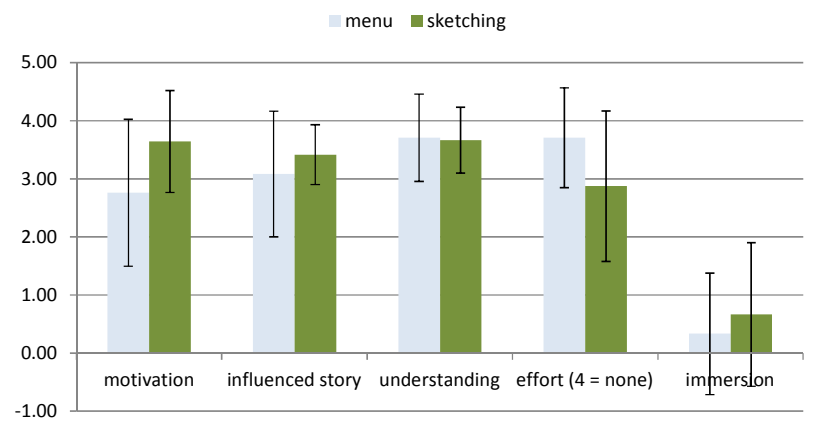

Fig. 8. Averages and standard deviation of questionnaire topics in both versions of the system 
Although the quantitative results are inconclusive, the increased motivation and interest in influencing the story, especially expressed in the interviews, indicate that this is a promising direction of research.

\section{Conclusion}

In this paper, we presented a mixed reality interactive storytelling system that allows users to visualize stories in augmented reality and to interact with virtual characters by sketching objects on a sheet of paper. As far as we are aware, this is the first time a pencil-and-paper interactive storytelling system is implemented and tested.

The storytelling approach presented in this paper opens up many possibilities to explore the user creativity, especially when we consider the use of our system as an education tool for children. As a future work, we intend to improve our system by including facilities to allow users, as much as possible, to extend the repertoire of recognizable sketches, and by designing an authoring tool to simplify and unify the authoring process in a single framework. We also intend to conduct more user studies with a larger number of participants to effectively validate our system.

\section{References}

1. Hagbi, N., Grasset, R., Bergig, O., Billinghurst, M., El-Sana, J.: In-Place Sketching for Content Authoring in Augmented Reality Games. In: Proceedings of IEEE Virtual Reality Conference 2010, Waltham, pp. 91-94 (2010)

2. Vogelsang, A., Signer, B.: The Lost Cosmonaut: An Interactive Narrative Environment on the Basis of Digitally Enhanced Paper. In: Proceedings of 3rd International Conference on Virtual Storytelling, Strasbourg, France, pp. 270-279 (2005)

3. Kuka, D., Elias, O., Martins, R., Lindinger, C., Pramböck, A., Jalsovec, A., Maresch, P., Hörtner, H., Brandl, P.: DEEP SPACE: High Resolution VR Platform for Multi-user Interactive Narratives. In: Proceedings of the 2nd Joint International Conference on Interactive Digital Storytelling, Guimarães, Portugal, pp. 185-196 (2009)

4. Bayon, V., Wilson, J.R., Stanton, D., Boltman, A.: Mixed reality storytelling environments. Virtual Reality 7(1), 54-63 (2003)

5. Dow, S., Mehta, M., Lausier, A., MacIntyre, B., Mateas, M.: Initial Lessons from ARFaçade, An Interactive Augmented Reality Drama. In: ACM SIGCHI Conference on Advances in Computer Entertainment, Los Angeles (2006)

6. Mateas, M.: Interactive Drama, Art, and Artificial Intelligence. Ph.D. Thesis - School of Computer Science, Carnegie Mellon University, Pittsburgh (2002)

7. Cavazza, M., Charles, F., Mead, S.J., Martin, O., Marichal, X., Nandi, A.: Multimodal acting in mixed reality interactive storytelling. IEEE Multimedia 11(3), 30-39 (2004)

8. Huynh, D.T., Raveendran, K., Xu, Y., Spreen, K., MacIntyre, B.: Art of Defense: A Collaborative Handheld Augmented Reality Board Game. In: Proceedings of the 2009 ACM SIGGRAPH Symposium on Video Games, pp. 135-142 (2009)

9. Leitner, J., Köffel, C., Haller, M.: Bridging the gap between real and virtual objects for tabletop game. International Journal of Virtual Reality 7(3), 1-5 (2009)

10. Ekman, P., Friesen, W.V.: Constants across cultures in the face and emotion. Journal of Personality and Social Psychology 17, 124-129 (1971)

11. Vapnik, V.: The Nature of Statistical Learning Theory. Springer, New York (1995)

12. El-Naqa, I., Yang, Y., Wernick, M.N., Galatsanos, N.P., Nishikawa, R.M.: A support vector machine approach for detection of microcalcifications. IEEE Transactions on Medical Imaging 21(12), 1552-1563 (2002) 
13. Cai, T., Hofmann, T.: Hierarchical document categorization with support vector machines. In: Proceedings of the 13th Conference on Information and Knowledge Management (2004)

14. Lima, E.S., Pozzer, C., Ornellas, M., Ciarlini, A., Feijó, B., Furtado, A.: Virtual Cinematography Director for Interactive Storytelling. In: Proceedings of the International Conference on Advances in Computer Entertainment Technology, Athens, Greece, pp. 263-270 (2009)

15. Hu, M.K.: Visual problem recognition by moment invariants. IRE Transactions on Information Theory 8, 179-187 (1962)

16. Zhou, Z., Cheok, A.D., Tedjokusumo, J., Omer, G.S.: wIzQubes ${ }^{\mathrm{TM}}$ - A Novel Tangible Interface for Interactive Storytelling in Mixed Reality. International Journal of Virtual Reality 7(4), 9-15 (2008)

17. Azuma, R., Baillot, Y., Behringer, R., Feiner, S., Julier, S., MacIntyre, B.: Recent Advances in Augmented Reality. IEEE Computer Graphics and Applications 21(6), 34-47 (2001)

18. Krevelen, D.W.F., van Poelman, R.: A survey of augmented reality technologies, applications and limitations. Int. J. of Virtual Reality 9(2), 1-20 (2010)

19. Jaimes, A., Sebe, N.: Multimodal Human-computer Interaction: A Survey. Computer Vision and Image Understanding 108(1-2), 116-134 (2007)

20. Malkawi, A., Srinivasan, R.: Multimodal human-computer interaction for immersive visualization: integrating speech-gesture recognitions and augmented reality for indoor environments. In: Proceedings of the Seventh IASTED Conference on Computer Graphics and Imaging, pp. 171-175. ACTA Press, Kauai (2004)

21. Dow, S., Mehta, M., Harmon, E., MacIntyre, B., Mateas, M.: Presence and Engagement in an Interactive Drama. In: Proc. of SIGCHI Conf. on Human Factors in Computing Systems (CHI 2007), San Jose, pp. 1475-1484 (2007)

22. Cavazza, M., Lugrin, J.-L., Pizzi, D., Charles, F.: Madame bovary on the holodeck: immersive interactive storytelling. In: Proc. of the 15th International Conf. on Multimedia (MULTIMEDIA 2007), pp. 651-660 (2007)

23. Igarashi, T., Zeleznik, B.: Sketch-based interaction - Guest Editors' Introduction. IEEE Computer Graphics and Applications 47(1), 26-27 (2007)

24. Sutherland, I.E.: Sketchpad: a man-machine graphical communication system. In: Proc. of AFIPS 1963, pp. 329-346. ACM, New York (1963)

25. Bergig, O., Hagbi, N., El-Sana, J., Billinghurst, M.: In-place 3D sketching for authoring and augmenting mechanical systems. In: Proc. of the 2009 8th IEEE Int. Symposium on Mixed and Augmented Reality (ISMAR 2009), pp. 87-94 (2009)

26. Cai, Y., Miao, C., Tan, A.-H., Shen, Z.: A hybrid of plot-based and character-based interactive storytelling. In: Hui, K.-c., Pan, Z., Chung, R.C.-k., Wang, C.C.L., Jin, X., Göbel, S., Li, E.C.-L. (eds.) EDUTAINMENT 2007. LNCS, vol. 4469, pp. 260-273. Springer, Heidelberg (2007)

27. Ciarlini, A.E.M., Pozzer, C.T., Furtado, A.L., Feijo, B.: A logic-based tool for interactive generation and dramatization of stories. In: Proc. of the ACM SIGCHI Int. Conf. on Advances in Computer Entertainment Technology, Valencia, pp. 133-140 (2005)

28. Ciarlini, A.E.M., Camanho, M.M., Doria, T.R., Furtado, A.L., Pozzer, C.T., Feijo, B.: Planning and interaction levels for TV Storytelling. In: 1st Joint Int. Conf. on Interactive Digital Storytelling, Erfurt, Germany, pp. 198-209 (2008)

29. Silva, F.G.A., Ciarlini, A.E.M., Siqueira, S.W.M.: Nondeterministic Planning for Generating Interactive Plots. In: Kuri-Morales, A., Simari, G.R. (eds.) IBERAMIA 2010. LNCS(LNAI), vol. 6433, pp. 133-143. Springer, Heidelberg (2010)

30. Cavazza, M., Charles, F., Mead, S.: Character-based interactive storytelling. IEEE Intelligent Systems, Special Issue on AI in Interactive Entertainment 17(4), 17-24 (2002) 